\title{
Myxoedema coma caused by immunotherapy-related thyroiditis and enteritis
}

\author{
Darran Mc Donald(101, Eirena Goulden1, Garret Cullen2, John Crown ${ }^{3,4}$ and \\ Rachel K Crowley1,4(1)
}

1Department of Endocrinology, 2Department of Gastroenterology, ${ }^{3}$ Department of Oncology, St Vincent's University Hospital, Dublin, Ireland, and ${ }^{4}$ Department of Medicine, University College Dublin, Dublin, Ireland
Correspondence should be addressed to D Mc Donald Email

darranmcdonald@gmail.com

\section{Summary}

Thyroid dysfunction is among the most common immune-related adverse reactions associated with immune checkpoint inhibitors. It most commonly manifests as painless thyroiditis followed by permanent hypothyroidism. This usually causes mild toxicity that does not interfere with oncological treatment. In rare instances, however, a life-threatening form of decompensated hypothyroidism called myxoedema coma may develop. We present a case of myxoedema coma in a woman in her sixties who was treated with a combination of CTLA-4 and PD-1 immune checkpoint inhibitors; for stage four malignant melanoma. She became hypothyroid and required thyroxine replacement after an episode of painless thyroiditis. Six months after the initial diagnosis of malignant melanoma, she presented to the emergency department with abdominal pain, profuse diarrhoea, lethargy and confusion. She was drowsy, hypotensive with a BP of $60 / 40 \mathrm{mmHg}$, hyponatraemic and hypoglycaemic. Thyroid function tests (TFTs) indicated profound hypothyroidism with a TSH of 19 $\mathrm{mIU} / \mathrm{L}$, and undetectable fT3 and fT4, despite the patient being compliant with thyroxine. She was diagnosed with a myxoedema coma caused by immune-related enteritis and subsequent thyroxine malabsorption. The patient was treated with i.v. triiodothyronine (T3) and methylprednisolone in the ICU. While her clinical status improved with T3 replacement, her enteritis was refractory to steroid therapy. A thyroxine absorption test confirmed persistent malabsorption. Attempts to revert to oral thyroxine were unsuccessful. Unfortunately, the patient's malignant melanoma progressed significantly and she passed away four months later. This is the first reported case of myxoedema coma that resulted from two distinct immune-related adverse reactions, namely painless thyroiditis and enterocolitis.

\section{Learning points:}

- Myxoedema coma, a severe form of decompensated hypothyroidism is a rare immunotherapy-related endocrinopathy.

- Myxedema coma should be treated with either i.v. triiodothyronine (T3) or i.v. thyroxine (T4).

- Intravenous glucocorticoids should be co-administered with thyroid hormone replacement to avoid precipitating an adrenal crisis.

- Thyroid function tests (TFTs) should be monitored closely in individuals with hypothyroidism and diarrhoea due to the risk of thyroxine malabsorption.

- A thyroxine absorption test can be used to confirm thyroxine malabsorption in individuals with persistent hypothyroidism. 


\section{Background}

Immune checkpoint inhibitors have revolutionised the treatment of oncologic malignancies over the past decade. They have been shown to improve progressionfree and overall survival in numerous cancers including malignant melanoma, non-small cell lung, renal cell and hepatocellular cancer (1). Additionally, a minority of patients with advanced malignancies can experience complete remission. Immune checkpoint inhibitors act by blocking intrinsic downregulators of immunity. Examples include the cytotoxic T-lymphocyte antigen 4 inhibitor (CTLA-4) ipilimumab and programmed-cell death 1 inhibitors (PD-1) nivolumab and pembrolizumab. They enhance the innate immune system's ability to detect and remove malignant cells. This novel mechanism of action also gives rise to the autoimmune side effects of immune checkpoint inhibitors which are commonly referred to as immune-related adverse effects. These reactions can affect any organ; however, the gastrointestinal, endocrine and hepatic systems are the most commonly affected.

Endocrinemanifestations include thyroiddysfunction, hypophysitis, adrenalitis, type 1 diabetes mellitus and hypoparathyroidism. Of these, hypothyroidism is the most common endocrinopathy with a prevalence of $1.5-6.8 \%$ with CTLA-4 inhibitors, $7-11 \%$ with PD-1 inhibitors and 4-27\% with combination therapy (2). Hypothyroidism typically follows an episode of painless thyroiditis and occurs on average 3 months after commencing treatment. It has been shown that the development of immune-related thyroid dysfunction from immune checkpoint inhibitors correlates with improved oncological progression-free and overall survival, compared to patients who do not develop this endocrinopathy (3). Hypothyroidism causes mild-moderate toxicity in almost all cases and does not interfere with oncological treatment. However, a lifethreatening form of decompensated hypothyroidism called myxoedema coma can occur. We present a unique case of myxoedema coma precipitated by two separate immunotherapy-related adverse reactions.

\section{Case presentation}

A woman presented to the emergency department with a 6-week history of worsening abdominal pain and profuse diarrhoea. She complained of fatigue, generalised weakness and had developed facial puffiness. On examination, she was hypotensive with a $\mathrm{BP}$ of $60 / 40 \mathrm{mmHg}$, heart rate of 88 b.p.m and temperature of $36^{\circ} \mathrm{C}$. She was drowsy but rousable to voice. Her abdomen was distended and mildly tender. It was also noted that the patient had periorbital oedema. She was hypoglycaemic with capillary blood glucose (CBG) of $3.9 \mathrm{mmol} / \mathrm{L}$.

She had been diagnosed with stage 4 malignant melanoma six months earlier and was treated with ipilimumab and nivolumab. The patient became hyperthyroid 6 weeks after commencing immunotherapy, with a TSH of $0.03 \mathrm{mIU} / \mathrm{L}(0.3-4.2)$, free T4 (fT4): 38.7 $\mathrm{pmol} / \mathrm{L}$ (11-22) and free T3 (fT3): $10.8 \mathrm{pmol} / \mathrm{L}$ (3.1-6.8). She then became hypothyroid one month later; TFTs revealed a TSH of $36.7 \mathrm{mIU} / \mathrm{L}$, fT4: $8.5 \mathrm{pmol} / \mathrm{L}$ and fT3: $2.5 \mathrm{pmol} / \mathrm{L}$. This pattern of hyperthyroidism followed by hypothyroidism is typical of a painless thyroiditis. She was commenced on T4 $75 \mu \mathrm{g}$ once daily. Follow-up TFTs had confirmed that this dose was appropriate. Immunotherapy was continued as this constituted a mild immunotherapyrelated adverse event.

\section{Investigation}

Routine admission bloods identified mild hyponatraemia with a sodium of $131 \mathrm{mmol} / \mathrm{L}$. TFTs were also performed given her clinical presentation and history of painless thyroiditis. They showed the patient was profoundly hypothyroid with a TSH of $19 \mathrm{mIU} / \mathrm{L}$, fT4 and fT3 were both undetectable. She was diagnosed with a myxedema coma based on clinical findings. In this case, the TSH of 19 $\mathrm{mIU} / \mathrm{L}$ was inappropriately low given an undetectable fT3 and fT4. It is important to note that TSH is not an accurate indicator of hypothyroidism severity in either myxedema coma or acutely unwell patients. A CT abdomen and pelvis demonstrated diffuse mucosal thickening of the small bowel, consistent with an enteritis (Fig. 1). The patient was diagnosed with an immunotherapy-related enteritis, thus predisposing them to thyroxine malabsorption and ultimately a myxedema coma.

\section{Treatment}

The patient was transferred to the ICU for inotropic support as her blood pressure failed to respond to aggressive fluid resuscitation. The hypothyroidism was treated with i.v. T3 5 $\mu \mathrm{g}$ TDS, and the enteritis treated with methylprednisolone. After receiving T3 replacement for $24 \mathrm{~h}$, the patient was alert, fully orientated and no longer required inotropic support. Her sodium and CBG also normalised. The T3 dose was titrated to $10 \mu \mathrm{g}$ TDS after several days to obtain a fT3 in the mid-normal range. The patient was discharged from the ICU after 5 days. 


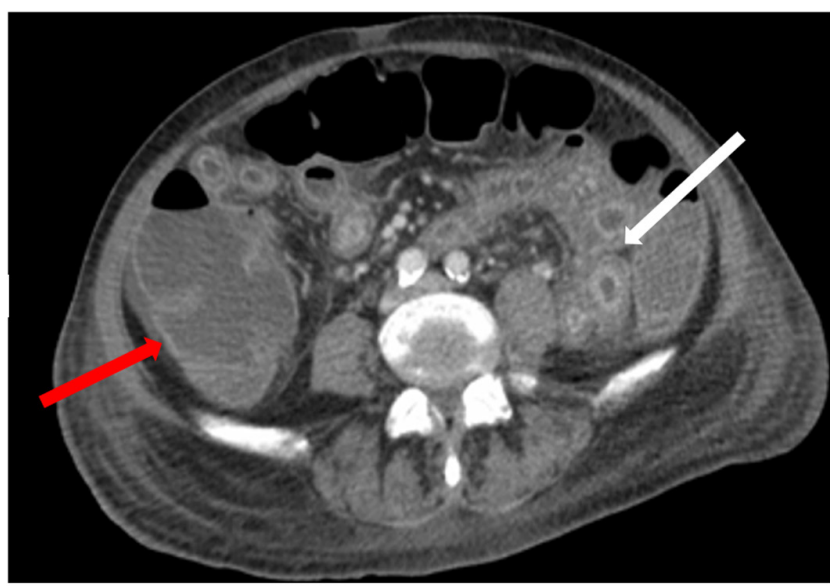

Figure 1

$\mathrm{CT}$ abdomen and pelvis demonstrating thickened loops of small bowel (white arrow) with peritoneal free fluid. Appearances of the caecum are normal (red arrow). The images were typical of enteritis.

\section{Outcome and follow-up}

She later underwent an OGD where duodenal biopsies were taken. These biopsies revealed acute on chronic duodenitis with extensive erosions and ulceration (Fig. 2). Although not specific, these findings were consistent with immunotherapy-related enteritis. Unfortunately, the enteritis was refractory to steroid therapy. A rapid thyroxine absorption test confirmed significant malabsorption. This test involved administering a stat of T4 $1000 \mu \mathrm{g}$ and monitoring TFTs hourly for $4 \mathrm{~h}$. Results showed a minimal increase in fT4 from 5 to $5.4 \mathrm{mmol} / \mathrm{L}$ and a reduction of fT3 from 2.8 to $2.3 \mathrm{pmol} / \mathrm{L}$ over the course of the test, thus confirming significant malabsorption (Table 1). Additionally, several attempts to transition the patient from i.v. T3 to oral T4 were unsuccessful as significant hypothyroidism ensued. Unfortunately, the patient's malignant melanoma progressed significantly and she died four months after her admission.

\section{Discussion}

This case outlines a life-threatening form of immunerelated hypothyroidism. Myxoedema coma is a form of decompensated hypothyroidism that is characterised by altered mental status, hypothermia, hypotension and hypoglycaemia. Decompensation into myxoedema coma occurs when a hypothyroid patient's homeostatic mechanisms are overwhelmed. Multiple factors may precipitate a myxoedema coma including infection, surgery, trauma or gastrointestinal haemorrhage. Myxoedema coma is a life-threatening form of hypothyroidism with a mortality rate ranging from

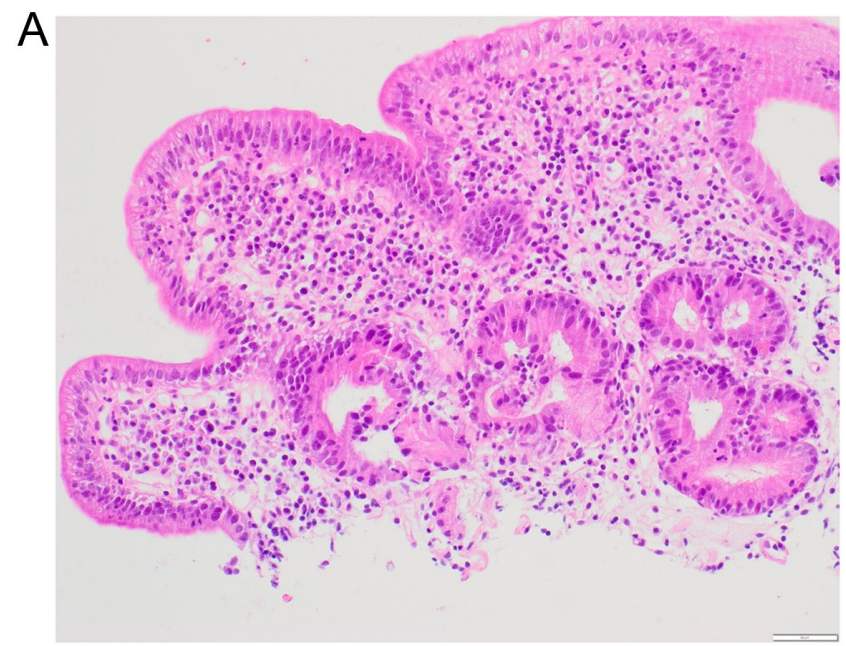

B

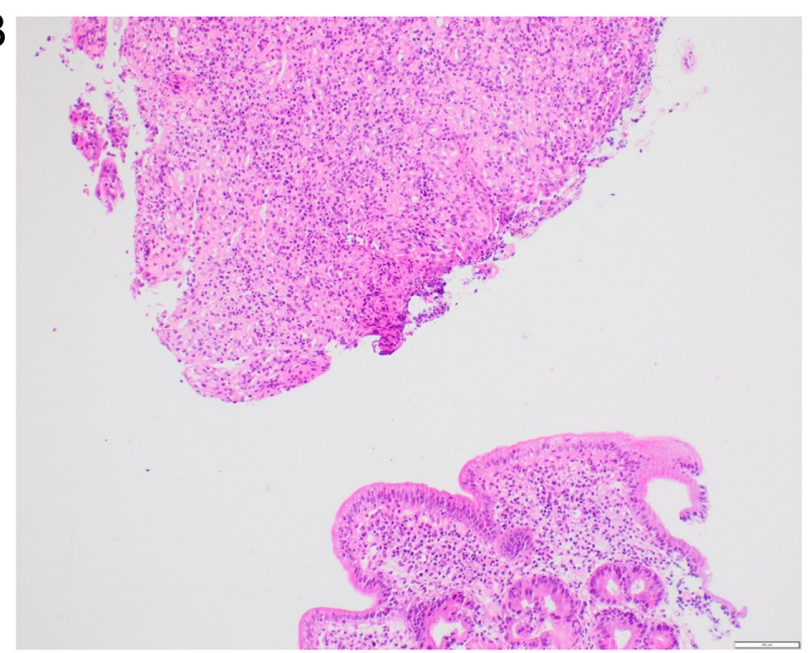

Figure 2

Duodenal biopsy. (A) Acute on chronic duodenitis with villous atrophy and infiltration of intraepithelial lymphocytes. (B) Extensive duodenal erosion and ulceration.

25 to $60 \%(4,5)$. Complications of myxoedema coma include cardiomyopathy and hypoventilation leading to respiratory failure. Affected patients should, therefore, be cared in an intensive care setting as inotropic support, mechanical ventilation and additional cardiac supports may be required.

Two other cases of myxoedema coma secondary to immune checkpoint inhibitor use have been reported in the literature to date $(6,7)$. Both cases occurred in patients with lung cancer, treated with PD-1 inhibitors, nivolumab and

Table 1 Results of thyroxine absorption test.

\begin{tabular}{|c|c|c|c|c|c|}
\hline & $0 \mathrm{~min}$ & $60 \mathrm{~min}$ & $120 \mathrm{~min}$ & $180 \mathrm{~min}$ & $240 \mathrm{~min}$ \\
\hline$\overline{\mathrm{TSH}}, \mathrm{mU} / \mathrm{L}$ & 0.16 & 0.18 & 0.19 & 0.21 & 0.26 \\
\hline fT4, pmol/L & 5 & 5.1 & 5.1 & 5.2 & 5.4 \\
\hline fT3, pmol/L & 2.8 & 2.7 & 2.5 & 2.5 & 2.3 \\
\hline
\end{tabular}


pembrolizumab. Myxoedema coma presumably followed an undetected episode of painless thyroiditis. Both patients had excellent responses to thyroid replacement. Our case has several unique features. It is the first case of myxoedema coma in a patient treated with a combination of CTLA-4 and PD-1 inhibitors. It is also the first case of myxoedema coma to have resulted from a combination of two distinct immune-related adverse effects, namely, painless thyroiditis and enterocolitis causing thyroxine malabsorption. The management of our patient was significantly complicated by a refractory enteritis and persistent malabsorption. As a result, our patient had a much poorer outcome. It is recommended that all patients taking immune checkpoint inhibitors should have baseline TFTs prior to commencing treatment, before each cycle and if symptoms of thyroid dysfunction occur (2). The presence of thyroid peroxidase and thyroglobulin antibodies at baseline may help clinicians identify those who are at higher risk of developing immune-related thyroid dysfunction.

Thyroid hormone replacement in the form of $\mathrm{T} 4$ or $\mathrm{T} 3$ is indicated in all cases of myxoedema coma. Treatment should be administered intravenously due to the risk of malabsorption. T4 must be metabolised to $\mathrm{T} 3$ via the enzyme type 1 deioiodinase (DIO1) to exert its biological effects. This enzyme is down regulated by acute illness. Therefore, T3 has a theoretical advantage over T4 in myxoedema coma as its action is independent of DIO1 activity (8). Treatment with thyroid hormone replacement must balance the risks of precipitating a tachyarrhythmia or myocardial infarction with the necessity for correction of the underlying condition. The choice of initial dose is influenced by the patient's clinical condition and cardiovascular co-morbidities. The American Thyroid Association recommends an initial bolus of 5 to $20 \mu \mathrm{g}$ of $\mathrm{T} 3$, followed by a maintenance dose of 2.5-10 $\mu \mathrm{g}$ every 8 $\mathrm{h}$ (8). We adopted a cautious approach in our patient and chose to omit the bolus of T3 and slowly titrate the dose after several days. It is important to monitor TFTs daily in the acute phase. Once the patient has stabilised, oral T4 can be commenced and i.v. T3 weaned. This was not possible in our case due to the patient's concomitant refractory enteritis.

Patients with myxoedema coma may also have adrenal insufficiency. This is of particular importance in oncology patients as other immunotherapy-related endocrinopathies include adrenalitis and hypophysitis which cause primary and secondary adrenal insufficiency, respectively. Furthermore, treatment with $\mathrm{T} 3$ decreases the activity of the enzyme 11ß-hydroxysteroid dehydrogenase type 1 (HSD11B1) which can result in decreased tissue exposure to cortisol (9). Treatment of a myxoedema coma with T3 can, therefore, precipitate an adrenal crisis. All patients with myxoedema coma should be treated with stress dose i.v. steroids in the acute setting. Adrenal reserve should be assessed prior to discontinuing steroids, once the patient is clinically improved.

The case highlights the use of a thyroxine absorption test. It is designed to differentiate malabsorption from pseudo-malabsorption in patients with apparent resistance to levothyroxine. There are two forms of the test in clinical use; the rapid and the long absorption test. Both require the direct observation of medication administration and monitoring for $60 \mathrm{~min}$ after. The rapid test involves administering T4 $1000 \mu \mathrm{g}$ and measuring TSH at 0, 1 and $2 \mathrm{~h}$ and fT 4 at $0,30,45,60,120$ and $240 \mathrm{~min}$. The long absorption test involves administering a dose of $1.6 \mu \mathrm{g} /$ $\mathrm{kg} /$ day on weekly basis for 4 weeks. TSH and fT 4 at 0,1 , 2, 3 and $4 \mathrm{~h}$ after ingestion on the first week and TSH and fT4 measured on the fifth week. There are no specific cutoffs for a positive test which limits the test's usefulness in clinical practice. However, the result clearly confirmed malabsorption in our patient. The rapid test was performed in this instance as our patient was hospitalised, meaning it was not possible to conduct the long version of the test over 4 weeks.

Malabsorption is an important differential in individuals who remain hypothyroid despite receiving a standard dose of T4 (greater than $1.6 \mu \mathrm{g} / \mathrm{kg}$ ). To determine the cause, a patient's compliance, administration routine and pregnancy status should be ascertained. Secondly, medications should be reviewed. PPIs and antacids alter gastric $\mathrm{pH}$ while iron and calcium supplementation or phosphate binders directly impair thyroxine absorption. Thereafter, symptoms and signs of malabsorption should be elicited. Autoimmune conditions such as coeliac disease and atrophic gastritis are more common in patients with hypothyroidism. These can be screened for by measuring IgA-tissue transglutaminase (TTG) and intrinsic factor antibodies, respectively. More detailed testing including breath tests for Helicobacter pylori or small bowel bacterial overgrowth may be performed. Finally, an OGD is usually required prior to making a definitive diagnosis of malabsorption (10).

There is a growing understanding of the range of immune-related adverse reactions caused by immune checkpoint inhibitors. We describe a case of myxoedema coma that arose from a unique combination of immunotherapy-related painless thyroiditis followed by enteritis causing thyroxine malabsorption. This case highlights the need for close monitoring of TFTs in those 
receiving immune checkpoint inhibitors given the high prevalence of thyroid dysfunction. A high index of suspicion is required to diagnose myxoedema coma in acutely unwell patients. Prompt diagnosis of this condition will allow clinicians to initiate potentially lifesaving i.v. thyroid hormone and steroid replacement.

\section{Declaration of interest}

The authors declare that there is no conflict of interest that could be perceived as prejudicing the impartiality of the research reported.

\section{Funding}

This work did not receive any specific grant from any funding agency in the public, commercial, or not-for-profit sector.

\section{Patient consent}

The patient's consent was obtained prior to her death.

\section{Author contribution statement}

All authors were involved in the clinical care of the patient and contributed to the writing of this manuscript.

\section{References}

1 Pan C, Liu H, Robins E, Song W, Liu D, Li Z \& Zheng L. Next-generation immuno-oncology agents: current momentum shifts in cancer immunotherapy. Journal of Hematology and Oncology 20201329. (https://doi.org/10.1186/s13045-020-00862-w)

2 Chang L-S, Barroso-Sousa R, Tolaney SM, Hodi FS, Kaiser UB \& Min L. Endocrine toxicity of cancer immunotherapy targeting immune checkpoint. Endocrine reviews 201940 17-65. (https://doi.org/10.1210/ er.2018-00006)

3 Basak EA, van der Meer JWM, Hurkmans DP, Schreurs MWJ, Oomen-de Hoop E, van der Veldt AAM, Bins S, Joosse A, Koolen SLW, Debets R, et al. Overt thyroid dysfunction and anti-thyroid antibodies predict response to anti-PD-1 immunotherapy in cancer patients. Thyroid 202030 966-973. (https://doi.org/10.1089/thy.2019.0726)

4 Jordan RM. Myxedema coma. Pathophysiology, therapy, and factors affecting prognosis. Medical Clinics of North America 199579185 - 194. (https://doi.org/10.1016/s0025-7125(16)30091-8)

5 Yamamoto T, Fukuyama J \& Fujiyoshi A. Factors associated with mortality of myxedema coma: report of eight cases and literature survey. Thyroid 19999 1167-1174. (https://doi.org/10.1089/ thy.1999.9.1167)

6 Khan UJR, Rizvi H, Sano D, Chiu J, Hadid T. Myxoedema crisis: case reportNivolumab induced myxedema crisis. Journal for immunotherapy of cancer 20175 13. (https://doi.org/10.1186/s40425-017-0213-x)

7 Gummalla S, Manjunath M \& Phillips B. Myxedema coma: a lifethreatening condition in patients using pembrolizumab. Case Reports in Endocrinology 202020208855943.

8 Garber JR, Cobin RH, Gharib H, Hennessey JV, Klein I, Mechanick JI, Pessah-Pollack R, Singer PA \& Woeber for the American Association KA. Clinical practice guidelines for hypothyroidism in adults: cosponsored by the American Association of Clinical Endocrinologists and the American Thyroid Association. Thyroid 2012 22 1200-1235. (https://doi.org/10.1089/thy.2012.0205)

9 Tomlinson JW \& Stewart PM. Cortisol metabolism and the role of 11ß-hydroxysteroid dehydrogenase. Best Practice and Research Clinical Endocrinology and Metabolism 200115 61-78.

10 Virili C, Antonelli A, Santaguida MG, Benvenga S \& Centanni M. Gastrointestinal malabsorption of thyroxine. Endocrine Reviews 2019 40 118-136. (https://doi.org/10.1210/er.2018-00168)
Received in final form 9 September 2021

Accepted 16 September 2021 